\title{
X-ray Structure of the R69D Phosphatidylinositol-Specific Phospholipase C Enzyme: Insight into the Role of Calcium and Surrounding Amino Acids in Active Site Geometry and Catalysis
}

\author{
David Apiyo, ${ }^{\ddagger}$ Li Zhao, ${ }^{\S}$ Ming-Daw Tsai, ${ }^{\S}$ and Thomas L. Selby*, \\ Department of Chemistry, The University of Central Florida, Orlando, Florida 32816, and Department of Chemistry, \\ The Ohio State University, Columbus, Ohio 43210
}

Received September 28, 2004; Revised Manuscript Received May 20, 2005

\begin{abstract}
Phosphatidylinositol-specific phospholipase Cs (PLCs) are a family of phosphodiesterases that catalyze the cleavage of the $\mathrm{P}-\mathrm{O}$ bond via transesterification using the internal hydroxyl group of the substrate as a nucleophile, generating the five-membered cyclic inositol phosphate as an intermediate or product. To better understand the role of calcium in the catalytic mechanism of PLCs, we have determined the X-ray crystal structure of an engineered PLC enzyme from Bacillus thurigiensis to $2.1 \AA$ resolution. The active site of this enzyme has been altered by substituting the catalytic arginine with an aspartate at position 69 (R69D). This single-amino acid substitution converted a metal-independent, low-molecular weight enzyme into a metal ion-dependent bacterial PLC with an active site architecture similar to that of the larger metal ion-dependent mammalian PLC. The $\mathrm{Ca}^{2+}$ ion shows a distorted square planar geometry in the active site that allows for efficient substrate binding and transition state stabilization during catalysis. Additional changes in the positions of the catalytic general acid/general base (GA/GB) were also observed, indicating the interrelation of the intricate hydrogen bonding network involved in stabilizing the active site amino acids. The functional information provided by this X-ray structure now allows for a better understanding of the catalytic mechanism, including stereochemical effects and substrate interactions, which facilitates better inhibitor design and sheds light on the possibilities of understanding how protein evolution might have occurred across this enzyme family.
\end{abstract}

Phosphatidylinositol-specific phospholipase C (PI-PLC) ${ }^{1}$ enzymes (EC 3.1.4.11) are important enzymes involved in various cellular signaling cascades that originate from the binding of hormones, neurotransmitters, and growth factors to specific extracellular receptors. Regulation of this class of enzymes has recently been reviewed (1). These phosphodiesterase enzymes catalyze the cleavage of the $\mathrm{P}-\mathrm{O}$ bond via transesterification using the axial hydroxyl group on the inositol ring as a nucleophile generating a fivemembered cyclic phosphate as an intermediate or as a final product as shown in Scheme $1(1-5)$. Schematically, the reaction mechanism appears to be straightforward, requiring a general acid-base system to facilitate proton transfer events and a positively charged species central to the acid-base pair to promote formation of the transition state phosphate species. However, the structural effects on kinetic rates of PI-PLCs are not easily understood for a number of reasons, demonstrated by the variability in releasing the cyclic or linear product, which is known to occur for bacterial PIPLC enzymes as well as mammalian isozymes that release

* To whom correspondence should be addressed. Telephone: (407) 823-6752. E-mail: tselby@mail.ucf.edu.

$\doteqdot$ The University of Central Florida.

$\S$ The Ohio State University.

${ }_{1}$ Abbreviations: 1,4,5-IP3, inositol 1,4,5-trisphosphate; 2,4,5-IP3, inositol 2,4,5-trisphosphate; cIP3, cyclic inositol trisphosphate; PI-PLC, phosphatidylinositol-specific phospholipase $\mathrm{C}$; rmsd, root mean-square deviation; WT, wild type.
Scheme 1: Reaction of PI-PLC in the R69D Active Site Utilizing the General Acid-General Base Histidine Residues (1), Which Are Required To Be Precisely Positioned for Efficient Catalysis $^{a}$
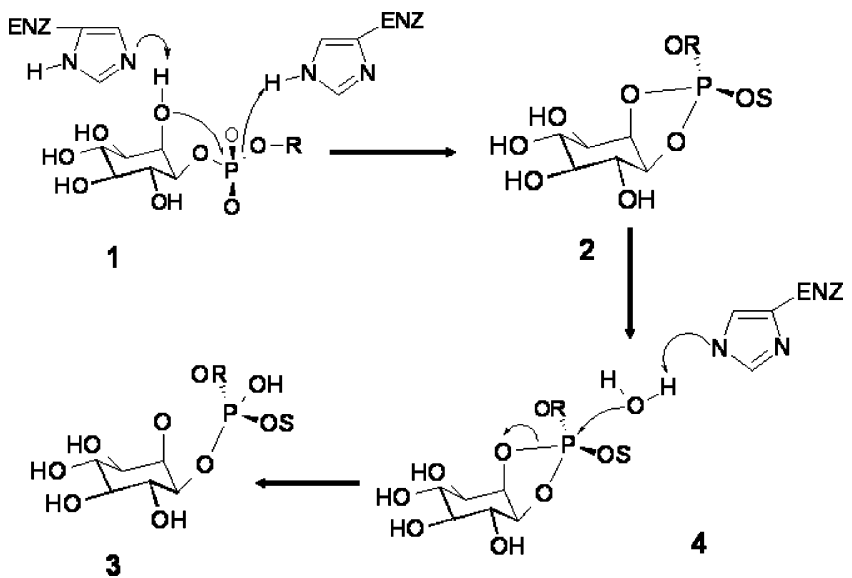

${ }^{a} \mathrm{The}^{2+} \mathrm{Ca}^{2+}$ ion (not shown) is involved in stabilizing the phosphonate formation by interacting with the oxygens $\left(\mathrm{O}_{R}\right.$ and $\left.\mathrm{O}_{S}\right)$ during formation of intermediate (2). The reaction is completed by hydrolysis (3) and finally product formation (4).

different amounts of cyclic phosphate upon their stimulation with an agonist-receptor mechanism (6). Mammalian PIPLCs are a diverse class of enzymes and represent a complex organization of structural domains, such as the pleckstrin homology (PH), EF-hand, C2, and conserved PLC-X and 
PLC-Y domains (3). The crystal structures of these domains have been determined individually [PDB entries 1DJX (7, 8), 1HSQ (9), 1MAI (10), and 1QAS (11)] and have provided a clearer understanding of the complexity and regulation of this enzyme family. Mammalian PI-PLC enzymes are also subject to scooting kinetics, and effects of interactions of their various structural domains with the lipid-water interface during catalysis. These complexities in the enzyme domain organization and kinetic attributes make the regulation of the various catalytic steps difficult to conceptualize based on the known chemical mechanism. In addition to these kinetic and domain differences, the mammalian enzymes are known to use a $\mathrm{Ca}^{2+}$ cofactor in their active sites to accelerate the reaction, whereas the smaller and less complex bacterial enzymes utilize a guanidinium group provided by an active site arginine residue. Because of the complexities in understanding the larger multidomain mammalian enzymes, the bacterial PI-PLCs have become an excellent model system for kinetic and structural investigations. These smaller bacterial enzymes, which have only one domain, have proven to be useful in structural characterization and have added to our understanding of kinetic changes based on active site amino acid substitutions $(12,13)$. However, as research on this initial model system has progressed, so has the need for better model systems that more closely resemble the mammalian enzymes for use in the design of therapeutics.

While the structure and mechanism of catalysis for the $\mathrm{Ca}^{2+}$-independent bacterial enzymes have been studied well, the specific role of the $\mathrm{Ca}^{2+}$ in the kinetics of the mammalian enzymes has been more difficult to explore because of the complex nature, and multiple metal binding sites involved in the mammalian enzyme's regulation as described above $(4,5)$. In addition, the question of enzyme adaptation or evolution comes into play when one considers the development of a higher-order mammalian enzyme from a simpler bacterial counterpart. In a comparison of the differences between the bacterial and mammalian enzymes, the use of $\mathrm{Ca}^{2+}$ in place of the active site arginine represents the simplest and most directly comparable variable that can be changed to better understand the changes toward a mammalian PI-PLC active site architecture as it relates to the enzyme's kinetic parameters. This change to $\mathrm{Ca}^{2+}$ allows a "mammalian" type active site to be constructed in a "bacterial" system to facilitate the understanding of the role of the $\mathrm{Ca}^{2+}$ and its effects on the kinetic rate and stereochemistry and how these relate to the active site geometry. Recently, Kravchuk et al. (14) demonstrated that a single-amino acid substitution of arginine at position 69 to aspartate (hereafter termed R69D) in the active site allowed a $\mathrm{Ca}^{2+}$-dependent bacterial enzyme to be successfully engineered.

The R69D enzyme exhibited interesting kinetic parameters, including a 30-fold rate enhancement upon the addition of $1 \mathrm{mM} \mathrm{Ca}^{2+}$. This enzyme had an overall similar structure relative to the wild-type (WT) protein based on NMR chemical shift values, but also demonstrated local changes in the active site amino acids upon the addition of $\mathrm{Ca}^{2+}$. The enzyme did exhibit a lower specific activity than the WT enzyme (in the presence of $\mathrm{Ca}^{2+}$ ), but had an increased stereoselectivity as shown by thio effects using DPPsI (15). The R69D enzyme without $\mathrm{Ca}^{2+}$ demonstrated a preference for sulfur in the pro- $R$ position, but this effect was enhanced more than 35 -fold by the addition of $\mathrm{Ca}^{2+}$. To better understand how the structure of this enzyme provides an active site environment to support these kinetic and mechanistic differences, we initiated crystal structural studies of the R69D enzyme and have determined the X-ray crystal structure of R69D at $2.1 \AA$ resolution.

\section{MATERIALS AND METHODS}

Crystal Growth and Optimization. The R69D protein from Bacillus thuringiensis was purified as described previously $(16,17)$. Crystals of R69D were originally found in a PEG ion screen (Hampton Research Inc., Aliso Viejo, CA) which contained 30\% PEG-3000, $0.2 \mathrm{M} \mathrm{NH}_{4} \mathrm{Cl}$, and $20 \mathrm{mM}$ CHES buffer ( $\mathrm{pH}$ 9.5). Crystals were grown at $4{ }^{\circ} \mathrm{C}$ using the hanging drop vapor diffusion method in standard Linbro plates (Hampton Research). Drops were set up using $1 \mu \mathrm{L}$ of protein $(25 \mathrm{mg} / \mathrm{mL}$ concentration) and $1 \mu \mathrm{L}$ of mother liquor. These conditions were then optimized using a grid array and varying the percentage of PEG $(25-35 \%)$ and $\mathrm{NH}_{4} \mathrm{Cl}(0.15-0.25 \mathrm{M})$ with $0.01 \mathrm{mM} \mathrm{CaCl} 2$ added to the mother liquor. All chemicals used for crystallization were purchased from Sigma Chemical Co. The largest crystals from each grid plate varied with each preparation, but were usually found in the range of 25-32\% PEG and $0.2 \mathrm{M} \mathrm{NH}_{4^{-}}$ $\mathrm{Cl}$. Hexagonal crystals measuring $0.10 \mathrm{~mm}$ on the longest edge were typically observed after 1 week. We harvested and cryoprotected crystals by soaking each crystal in PEG400 for $5-10 \mathrm{~s}$ before freezing them in liquid $\mathrm{N}_{2}$ prior to data collection.

Data Collection and Processing. Data were collected at the Advanced Light Source (ALS) beamline 5.0.1 using a 2 $\times 2 \mathrm{CCD}$ array (ADSCQ4U) detector at $100 \mathrm{~K}$. Data were indexed, scaled, and integrated using the HKL 2000 software package in space group $C 222$ with the following cell dimensions: $a=91.2 \AA, b=147.7 \AA, c=96.5 \AA$, and $\alpha$ $=\beta=\gamma=90^{\circ}(18)$. After the data had been indexed and scaled, systematic absences were observed in the log files, and the space group was determined to be $C 222_{1}$. Data statistics are listed in Table 1.

Structure Determination and Refinement. A molecular replacement (MR) solution was successfully found using MOLREP as part of the CCP4 software suite $(19,20)$ using the PI-PLC enzyme from Bacillus cereus (PDB entry 1PTG) (21). The TF/sig value was 45.33 with an $R$ of $52.1 \%$ and a correlation factor of $35.7 \%$. This initial model ( $R$ and $R_{\text {free }}$ values of 43 and $48 \%$, respectively) was then subjected to rigid body refinement followed by simulated annealing using the CNS software package (22). These manipulations resulted in $R$ and $R_{\text {free }}$ values of 35 and $40 \%$, respectively. This model was then further refined using a combination of automated REFMAC runs and manual builds using XtalView and $\mathrm{O}$ $(23,24)$. Following refinement of the polypeptide model to $R$ and $R_{\text {free }}$ values of 24 and $29 \%$, respectively, water positions were found using CNS and $\operatorname{ARP} / w \operatorname{ARP}(22,25)$. Following the determination of water positions, the $\sigma$ level in the electron density maps was increased to $8 \sigma$ to show the locations of the $\mathrm{Ca}^{2+}$ ions. $\mathrm{Ca}^{2+}$ ion positions were then verified using the surrounding amino acids as a guide to ensure proper molecular geometry for the $\mathrm{Ca}^{2+}$ ion. Final geometrical restraints were analyzed using PROCHECK $(26$, 27). The final refined structure has been deposited as PDB entry $1 \mathrm{~T} 6 \mathrm{M}$ and RCSB entry 022377. 


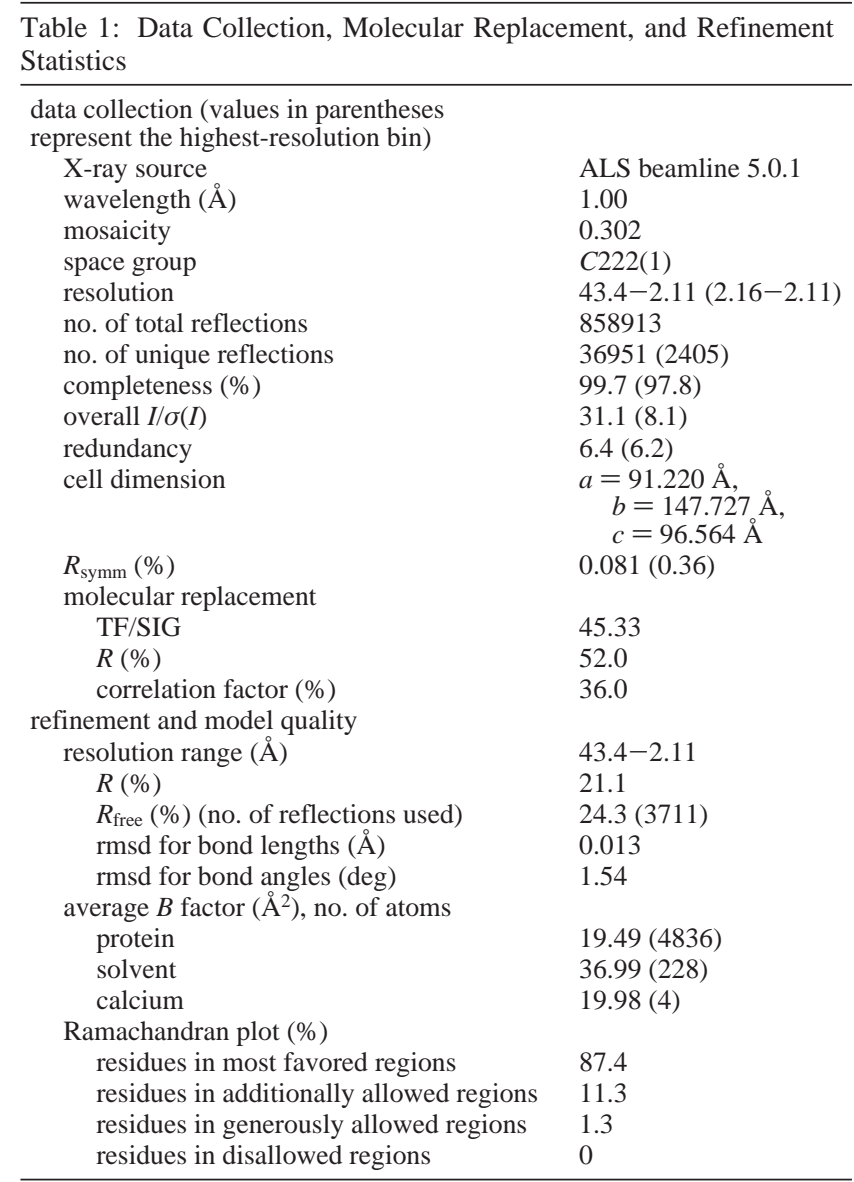

Table 2: Active Site Interatomic Distances for R69D

\begin{tabular}{clc}
\multicolumn{3}{c}{ Calcium Coordination } \\
\hline atom 1 & atom 2 & distance $(\AA)$ \\
\hline Asp 33 OD2 & $\mathrm{Ca}^{2+}$ & 2.1 \\
Asp 69 OD1 Ca & $\mathrm{Ca}^{2+}$ & 2.1 \\
HOH 1 Ca & $\mathrm{Ca}^{2+}$ & 3.2 \\
HOH 3 Ca & $\mathrm{Ca}^{2+}$ & 3.6 \\
HOH 112 Ca & $\mathrm{Ca}^{2+}$ & 3.7 \\
Asp 67 OD2 & $\mathrm{Ca}^{2+}$ & 3.8
\end{tabular}

Potential Interactions with Inositol and Cyclic Inositol

\begin{tabular}{|c|c|c|}
\hline \multicolumn{3}{|l|}{ inositol } \\
\hline $\mathrm{O} 2$ (axial oxygen on $\mathrm{C} 2$ ) & $\mathrm{Ca}^{2+}$ & $\begin{array}{l}3.3 \text { (previously occupied } \\
\text { by } \mathrm{HOH} 3 \text { ) }\end{array}$ \\
\hline \multicolumn{3}{|l|}{ cyclic inositol } \\
\hline $\mathrm{O} 2$ & $\mathrm{Ca}^{2+}$ & 3.7 \\
\hline $\mathrm{O} 1 \mathrm{P}\left(\mathrm{O}_{R}\right)$ & $\mathrm{Ca}^{2+}$ & $\begin{array}{l}2.9 \text { (previously occupied } \\
\text { by } \mathrm{HOH} 1 \text { ) }\end{array}$ \\
\hline $\mathrm{O} 2 \mathrm{P}\left(\mathrm{O}_{S}\right)$ & $\mathrm{Ca}^{2+}$ & 1.9 \\
\hline
\end{tabular}

Inositol (Ins) and Cyclic Inositol (cIns) Overlay Models. The experimentally determined R69D structure with inositol modeled into the active site was created by overlaying the PI-PLC enzyme from B. cereus (PDB entry $1 \mathrm{PTG}$ ), which contained inositol in the active site, on top of the R69D structure using the best fit $\alpha$-carbon superpositioning as determined by the TOP program as part of the CCP4 software suite $(20,28)$. Following the creation of the R69D-Ins model, the cyclic inositol model was created by overlaying the ring system from the cyclic inositol onto the ring system of the bound inositol compound. No changes were made to the R69D polypeptide structure. This manipulation resulted in the R69D-cIns model. These overlayed models showed various hydroxyl groups (from Ins and cIns) superpositioned with enzyme active site waters. In cases where the experimentally determined water positions showed superpositioning within $1 \AA$ with inositol hydroxyl groups (see the Supporting Information), the experimentally determined waters were deleted and analysis of the interaction of the hydroxyl group with the enzyme active site was completed as described in the next section. It is important to note that all water positions represented in the figures showing the R69D active site structure were experimentally determined and not from the 1PTG coordinate overlays or from the mammalian structure.

Energy Optimization. Molecular modeling studies of R69D's interaction with various inositol substrates were carried out using BiomedCache version 6.1 (Fujitsu Inc.). The structures of PI-PLC from B. cereus (1PTG) and mammalian PI-PLC (1DJX) were obtained from the Protein Data Bank (PDB) and used for comparison purposes. Energy optimization was done using molecular mechanics 3 (MM3) force field calculations. The enzymes were kept rigid except for the side chains of Asp 33, Asp 67, Glu 117, and Arg 69/Asp 69 in 1PTG and R69D and Asn 312, Glu 341, Asp 343, and Glu 390 from the mammalian structure.

\section{RESULTS}

Crystal Structure. The R69D structure has excellent density along the entire polypeptide backbone except for the regions from amino acids $122-127$ and $240-242$. The electron density of the active site region is shown in Figure 1 . The final $R$ is $21.1 \%\left(R_{\text {free }}=24.3 \%\right)$, which is comparable to the 1PTG structure that had an $R$ of $19.5 \%$ and an $R_{\text {free }}$ of $24.3 \%$. When the R69D enzyme is overlayed with the 1 PTG structure, five regions that differ by a rmsd of nearly $1 \AA$ were found at the backbone. As shown in Figure 2, only one region (marked $\mathrm{E}$ on the plot) is in the vicinity of the active site (indicated by the calcium ion position as a yellow sphere). The other regions are outer loop regions of the protein and are more flexible. The regions marked D and $\mathrm{F}$ are polypeptide regions with poor electron density, and their comparisons to the 1PTG structure cannot be made accurately. Analysis of the Ramachandran plot (not shown) demonstrates that all but seven amino acids are located in the most favored or additionally allowed regions, and these amino acids are all located in the regions without density described above. The overall temperature value (mean $B$ value) of $19.49 \AA^{2}$ is quite similar to that of the 1 PTG structure of 19.5. However, one of the loop regions with poor electron density mentioned above (amino acids 244-246) had temperature values that were much lower $(\cong 42)$ than those of comparable regions in the 1PTG structure $(\cong 70)$, indicating a lower degree of flexibility as compared to the 1PTG (WT) enzyme. It should be noted that R69D and 1PTG have a high level of sequence identity $(96 \%)$ and that the eight different amino acids are far from the active site and in fact do not contribute to any structural differences.

Interestingly, with only eight different amino acid differences between the 1PTG structure and R69D, we find that 1PTG is crystallized in space group $P 2{ }_{1} 2_{1} 2_{1}$ with one molecule in the asymmetric unit and R69D crystallizes in space group $C 222_{1}$ with two molecules per asymmetric unit.

Active Site Architecture of R69D. The active site $\mathrm{Ca}^{2+}$ ion was found in the proximity of the region previously occupied 


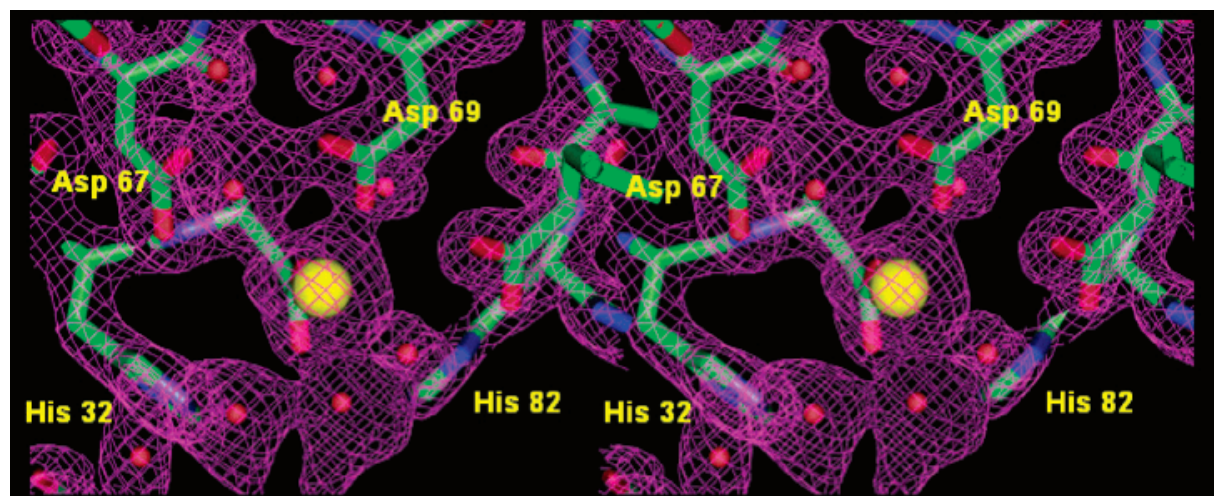

FIGURE 1: Stereoview of the difference $\left(2 F_{\mathrm{o}}-F_{\mathrm{c}}\right)$ map of the R69D active site region contoured to $1.5 \sigma$ showing the active site calcium ion (yellow) and the coordinated active site water molecules (red). Chelating amino acid residues Asp 67 and Asp 69 are also shown as well as the general acid-base histidines (His 82 and His 32). The third chelating amino acid (Asp 33) is partially eclipsed by the Ca ${ }^{2+}$ ion.

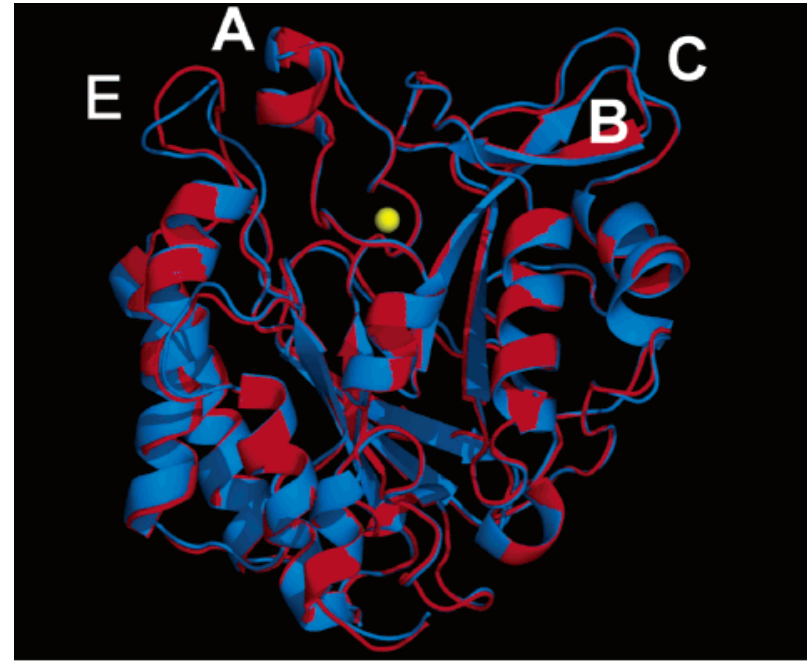

C-Alpha Variation: R690 vs Wild Type

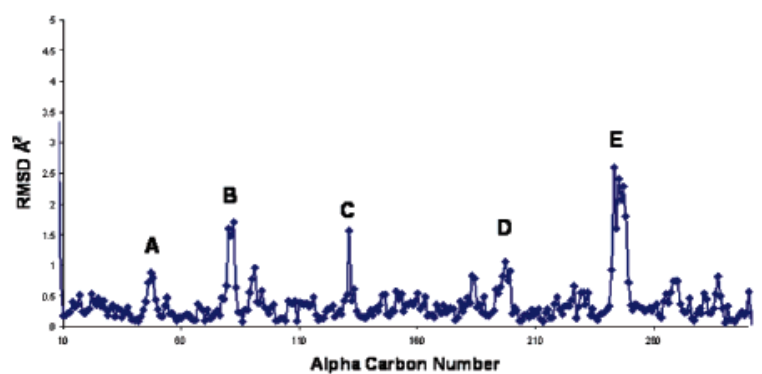

Figure 2: Backbone overlay of the R69D enzyme with the PIPLC enzyme from $B$. cereus (PDB entry 1 PTG) showing main chain regions that have moved by $\geq 1 \AA$ (not including the N-termini). The calcium ion is colored yellow as a reference point for the active site. Region D is located behind the structure and is not marked on the protein overlay.

by the arginine guanidinium group as predicted (14). The distance from $\mathrm{Ca}^{2+}$ to the central carbon $(\mathrm{Cz})$ of the guanidinium group measures $1.4 \AA$. The geometry around the calcium is a distorted square pyramidal system that utilizes the neighboring aspartate residues (Asp 33 and Asp 69) and a number of active site waters. A list of all active site amino acids and waters that are in the proximity of the calcium ion are given in the Supporting Information. Of all the atoms appearing in the table, only four are within proper bonding distances to be considered actual ligands in the calcium interaction. The additional amino acids are included for comparison with other PI-PLC structures and to show secondary interactions. The primary interacting amino acids
(Asp 33 and Asp 69) along with three water molecules form the primary interacting atoms, and the other atoms in the active site appear to contribute to an extended hydrogen bonding network that is formed due to the calcium ion being added, but are more than $3.5 \AA$ from the calcium. Considering hard-soft acid base chemistry effects, it is clear that the addition of a hard metal such as calcium into an active site that was previously occupied by a soft delocalized guanidinium group would promote stronger interaction with waters in the active site. This is demonstrated in our structure where ordered waters, with low $B$ values, are found in the active site. This appears to be required to allow for proper molecular geometry to be formed around the calcium ion with only two oxygen atoms from two different carboxylate groups, while maintaining an optimal hydrogen network with neighboring side chains in the active site. This is in contrast to the three waters that are found in the active site for the WT structure (PDB entry 1PTD) that form a different pattern of hydrogen bonds due to the availability of the hydrogen bond-donating guanidinium group.

The major difference in the catalytic general acid-base arrangement is seen in His 82 . The position of His 32 remains primarily unchanged, and it has the same hydrogen bonding arrangement with Asp 274 as in the WT structure (29). There is a slight change in rotation of the imidazole ring induced by hydrogen bonding to one of the calcium chelating waters. The His 82 side chain has been moved inward, providing a strong hydrogen bond to a second water molecule that is located in the active site, but not involved in the calcium interaction. In the WT structure, this histidine is involved in a catalytic triad arrangement with Arg 69 and Asp 33. With the Arg 69 side chain replaced with $\mathrm{Ca}^{2+}$ and the Asp 33 more closely involved with this ion, the catalytic triad has been significantly altered. This in turn disrupts the interaction of Asp 33 with His 82 and allows the altered position to occur, but does provide a hydrogen bond acceptor to interact with the active site water as described. Additionally, this histidine shows larger than average $B$ values $\left(25-28 \AA^{2}\right.$ ), indicating a higher degree of mobility as compared to other active site amino acids.

Also, significant movement near the loop region surrounding amino acids $80-86$ (see Figure 2) is observed. This demonstrates that the changes observed in the location of His 82 extend into the main chain region as well showing larger structural effects than anticipated in chelating the calcium ion. Overall, these changes result in a slightly more 


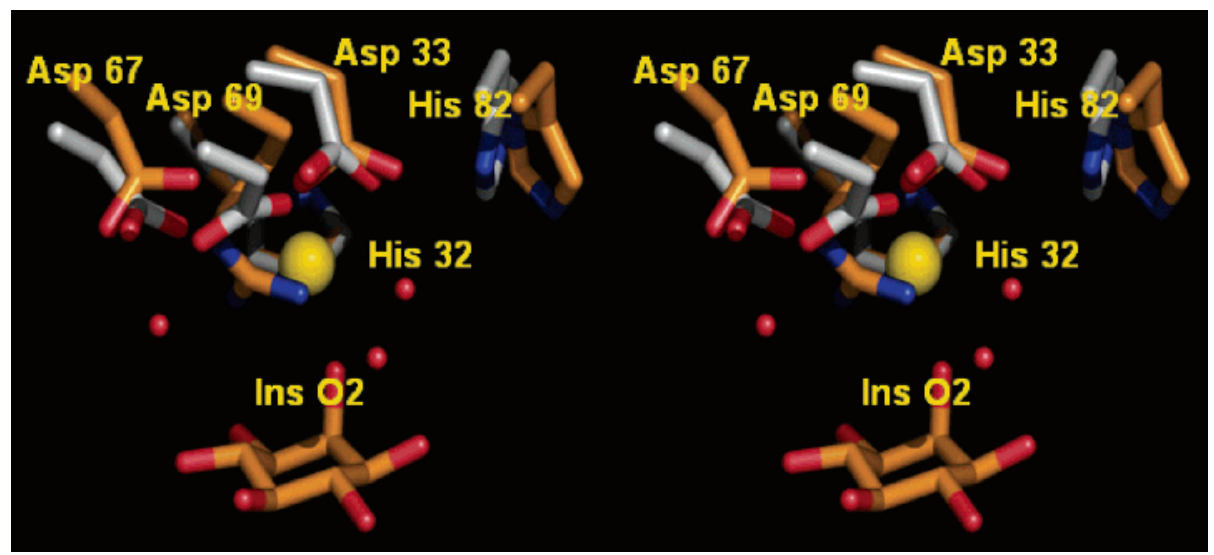

FIGURE 3: Stereoview of the side chain overlay of the active site amino acids from R69D and the PI-PLC enzyme from B. cereus (PDB entry 1PTG), showing changes in the active site amino acids upon aspartate replacement (or Arg 69) and $\mathrm{Ca}^{2+}$ addition. 1PTG is shown with orange carbons, and R69D is colored gray. Note the location of the $\mathrm{Ca}^{2+}$ relative to the previous position of the guanidinium group of Arg 69 in the active site. The inositol ring position was determined in the 1PTG structure and not modified in this overlay, and the water molecules (red spheres) were determined in the R69D structure. This diagram shows the rotation of His 86 creating a more closed active site as compared to that of the 1PTG structure.

closed active site compared to that of the WT enzyme and may contribute to the slightly reduced catalytic rate.

Comparison with the Mammalian Active Site. The crystal structure of mammalian PI-PLCs shows that the calcium is chelated to Asn 312 (OD1), Glu 341 (OE1), Asp 343 (OD1 and OD2), and Glu 390 (OE1). All of these residues have been shown to be important for catalysis as well as $\mathrm{Ca}^{2+}$ binding $(7,30)$. In the mammalian structure, a single water molecule is also bound to the calcium ion, completing the pentavalent coordination system. In comparing the R69D structure to the mammalian active site, we find two major differences. The first involves the number of interacting amino acids, and the second involves the type of interactions that each of the interacting amino acids makes with the calcium ion.

The mammalian active site shows an optimal geometrical arrangement of chelating amino acid side chains surrounding the $\mathrm{Ca}^{2+}$ that are both equidistant from one another to provide equivalent binding for the ion and equidistant from the ion itself. This type of binding allows the $\mathrm{Ca}^{2+}$ to alternate between the two binding geometries that have been described to occur during substrate binding (31). The mammalian system is known to undergo a transition from an octahedral geometry when $1,4,5$-IP3 is bound to a square pyramidal geometry when 2,4,5-IP3 is bound. During the binding of 1,4,5-IP3, the 2-hydroxyl group is the sixth ligand for the calcium and a water molecule interacts with the calcium to complete the geometry. When the 2,4,5-IP3 is bound, the water molecule is replaced with the OP2 atom of the 2-phosphoryl group. During the binding of the cyclic intermediate, the geometry is somewhat distorted, allowing both the phosphonate $\mathrm{O}_{S}$ and $\mathrm{O}_{R}$ atoms to bind to the calcium, and the 2-hydroxyl moves out of binding proximity. The authors report very little conformational change for each of these structures and describe "prestabilizing" interactions among active site residues. This is similar to the bacterial PI-PLC enzymes in which no significant conformational changes are observed upon inositol binding (21). Overall, the calcium interaction in the mammalian system uses five amino acids, and of those five, one has both oxygen atoms from its carboxylate functional group interacting with the calcium ion $(8,31)$. This geometry demonstrates an intricate, optimally arranged set of $\mathrm{Ca}^{2+}$-binding atoms that provides a well-ordered binding pocket that was not quite achieved in the R69D structure The R69D active site shows two interacting amino acids each binding the $\mathrm{Ca}^{2+}$ ion through one oxygen atom from their respective carboxylate groups as shown in Figure 3 . The molecular geometry surrounding the calcium is completed by a number of water molecules. The geometrical arrangement of atoms around the calcium ion is a distorted square pyramidal system, which is similar to the mammalian active site, but in R69D, the two chelating amino acids are at an angle of $90^{\circ}$ relative to each other. This certainly causes some weakening of the $\mathrm{Ca}^{2+}$ binding energy in the active site of the enzyme. Additionally, these aspartate amino acids bind the calcium with one oxygen atom from each carboxylate group. OD2 of Asp 67 is near the active site, but is too distant to interact with the $\mathrm{Ca}^{2+}$ ion. If this amino acid were capable of binding the $\mathrm{Ca}^{2+}$, creating a geometrical arrangement similar to that of the mammalian enzyme, there would be an opposing amino acid side chain on each side of the metal providing an optimal binding pocket for the calcium ion. It is obvious, based on the observation of $\mathrm{Ca}^{2+}$ being found in the R69D structure, that having two amino acids in the active site to bind the metal is enough to support the positioning of the ion. However, completing the molecular geometry of the metal with waters instead of amino acids could have an effect on the strength of interaction of the calcium with the protein and allow movement of the calcium position during the different steps of catalysis. Therefore, the weakened positioning for the engineered enzyme opens up the possibility for mobility of the calcium ion, which may be influencing different aspects of the kinetic mechanism through interactions of the inositol and phosphonate oxygen atoms.

When rate enhancement studies were completed for the bacterial WT, bacterial mutant, and mammalian enzymes, the $K_{\mathrm{d}}$ app for the R69D enzyme was found to be 180 and $160 \mu \mathrm{M}$ for calcium and magnesium, respectively (14). However, the mammalian enzyme binding constant was found to be $1.3 \mu \mathrm{M}$ for calcium and showed no rate enhancement with the addition of $\mathrm{Mg}^{2+}$ up to $5 \mathrm{mM}$ (32, 33). These metal ion affinity results are corroborated by this structural work, which demonstrates that the binding geom- 


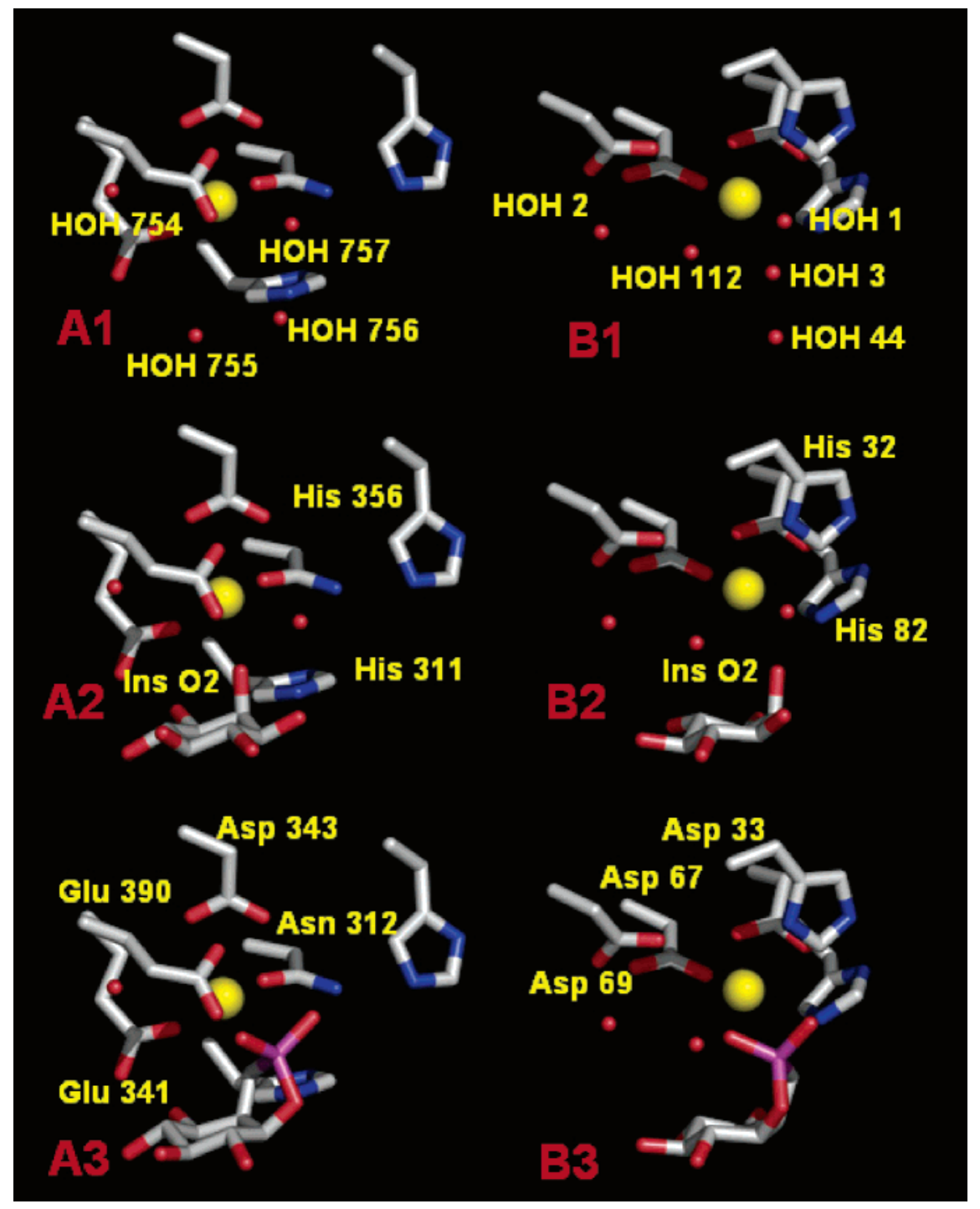

FIGURE 4: Comparison of the active sites of the mammalian PI-PLC with R69D demonstrating the different types of interactions in the mammalian calcium-dependent system as compared to the R69D enzyme with $\mathrm{Ca}^{2+}$ added: (top panel) comparison of R69D to PDB entry 1DJI showing similarities in active site water positions (with waters numbered), (middle panel) similarities in inositol binding (comparison with PDB entry 1DJX) with general acid-base histidines marked, and (bottom panel) similarities in binding of cyclic inositol (PDB entry 1DJY) with those amino acids chelating the $\mathrm{Ca}^{2+}$ labeled. Note that the water molecules coordinated in the top panel (HOH 3 in R69D and $\mathrm{HOH} 755$ in 1DJI) align within $0.5 \AA$ of the oxygen atoms from the inositol ring in the middle panel and $\mathrm{HOH} 1 \mathrm{in}$ R69D and $\mathrm{HOH} 757$ in $1 \mathrm{DJI}$ in the bottom panel.

etry of R69D, composed of only two amino acid interactions and surrounding active site water molecules, is much weaker than the interaction of the mammalian enzyme with five amino acid interactions. Additionally, the distorted square pyramidal geometry of the R69D active site will allow both $\mathrm{Ca}^{2+}$ and $\mathrm{Mg}^{2+}$ to occupy the active site with similar affinities, but the active site geometry in the mammalian system, which requires a transition from an octahedral state to a square pyramidal state, would not accommodate $\mathrm{Mg}^{2+}$ effectively.

To compare the interactions of R69D with inositol with those of the mammalian system, we overlaid the inositolbound structure of the WT with the R69D enzyme (see Materials and Methods). Analysis of the active site demonstrated that the $\mathrm{O} 2$ atom (2-hydroxyl on the inositol ring) overlays with one of the chelating water molecules to within $1 \AA$. This would indicate that the inositol binding position within the R69D structure is similar to that of the mammalian enzyme, which had the $\mathrm{O} 2$ atom in an identical position.
Our overlay analysis cannot determine the extent of any conformational changes that may occur in the enzyme due to binding inositol, but examination of the WT bacterial forms of the enzyme (with and without inositol present) shows no conformational changes upon binding $(21,34)$. Additionally, this binding event allows the other waters that are surrounding the calcium ion $\mathrm{HOH} 1, \mathrm{HOH} 2$, and $\mathrm{HOH}$ 112) to remain in place during modeling of the inositol ring into the active site as shown in Figure 4. Therefore, not only is the interaction of the 2-hydroxyl position conserved between the mammalian and R69D enzymes, but the surrounding water molecule interactions also appear to have similarities.

Analysis of Cyclic Inositol (cIPs). Using the same method to overlay the cIP molecule demonstrated that the phosphonate $\mathrm{O}_{R}$ was within $1 \AA$ of one of the water molecules that was originally binding the calcium ion. This produced a geometry in which the $\mathrm{O}_{R}$ and $\mathrm{O}_{S}$ atoms of the phosphonate were 2.87 and $1.92 \AA$ from the $\mathrm{Ca}^{2+}$ ion, respectively. This 
is also similar to the binding of cIP in the mammalian structure with the primary difference being that the $\mathrm{O}_{R}$ and $\mathrm{O}_{S}$ atoms are 2.6 and $2.2 \AA$ from the $\mathrm{Ca}^{2+}$ ion, respectively. Given the degree of distortion from the ideal molecular geometry of the calcium ion in the R69D enzyme as compared to that in the mammalian system, it is conceivable that the differences in distances from the $\mathrm{Ca}^{2+}$ between the $\mathrm{O}_{R}$ and $\mathrm{O}_{S}$ atoms observed in the overlays are a good model and could help to explain the stereochemical effects of the R69D enzyme as compared to the WT. A panel showing the comparisons of these interactions appears in Figure 4. Although the active site residues from the mammalian and bacterial enzymes are not superimposable, it is interesting to observe the similarities in how the two different active sites bind the calcium ion, the surrounding water molecules, and the inositol compounds. The mammalian active site uses the 2-OH group of the inositol to complete the coordination for the octahedral geometry in the same manner as the R69D enzyme, even though the coordination geometries are not the same. Additionally, when the inostol is absent from the active site, tightly bound waters complete the coordination geometry (and are superimposable when modeled into the active site of the R69D structure) in a manner similar to that of the mammalian enzyme.

$R_{P} / S_{P}$ Stereoselectivity of WT and R69D-Ca $a^{2+}$ btPI-PLC. An earlier study by Kravchuk et al. showed that the correct positioning and the bidentate nature of Arg69 account for the extremely high $R_{\mathrm{P}} / S_{\mathrm{P}}$ stereoselectivity $\left(k_{R_{\mathrm{P}}} / k_{S_{\mathrm{P}}}=7600\right)$ conferred by the WT enzyme (15-17). These results suggested that the hydrogen bond interaction between the side chain of Arg69 and the pro- $S_{\mathrm{P}}$ oxygen of the substrate stabilizes the pentacovalent transition state.

A sulfur substitution at the $p r o-S_{\mathrm{P}}$ position weakens the hydrogen bond interaction and leads to a drastic decrease in the reaction rate $\left(S_{\mathrm{P}}\right.$ thio effect, $\left.k_{\mathrm{O}} / k_{S_{\mathrm{P}}}=3.1 \times 10^{5}\right)$ catalyzed by the WT enzyme. Experimentally, R69D in the absence of $\mathrm{Ca}^{2+}$ exhibited a 3 order of magnitude drop in the $S_{\mathrm{P}}$ thio effect $\left(k_{\mathrm{O}} / k_{S_{\mathrm{P}}}=7.8 \times 10^{2}\right)$, which was then restored to a proper level $\left(k_{\mathrm{O}} / k_{S_{\mathrm{P}}}=4.4 \times 10^{5}\right)$ upon $\mathrm{Ca}^{2+}$ addition. These suggest that the bound $\mathrm{Ca}^{2+}$ positions are similar to that of the Arg 69 side chain and mimic the bidentate nature of Arg 69 (14). To provide structural support to these interpretations, we performed molecular modeling studies on WT and R69D using various inositol phosphate analogues (details in Materials and Methods). Our results show that the $\mathrm{Ca}^{2+}$ cofactor and Arg 69 side chain indeed interact with the phosphate moiety in an extremely similar manner. Our results show that $\mathrm{Ca}^{2+} / \mathrm{Arg} 69$ forms a hydrogen bond with the pro- $S_{\mathrm{P}}$ oxygen of inositol 2-phosphate and inositol 1,2-cyclic phosphate. The distance between $\mathrm{Ca}^{2+} / \mathrm{Arg} 69$ and the pro$S_{\mathrm{P}}$ oxygen of the substrate analogues is within $3 \AA$ (see Supporting Information). Although the interaction between $\mathrm{Ca}^{2+} /$ Arg 69 and the pro- $S_{\mathrm{P}}$ oxygen of the product intermediate, inositol 1,2-cyclic phosphate, is analogous to that in mammalian PI-PLC, the product intermediate is readily released from the active site of the enzyme in the btPI-PLCcatalyzed reaction. A feasible explanation is discussed in the context of the mammalian PI-PLC (in complex with the product intermediate) in the next section.

The pro- $R_{\mathrm{P}}$ oxygen does not readily participate in catalysis; however, a sulfur substitution at this position likely introduces an unfavorable interaction of the sulfur atom with another part of the active site, and consequently, a $R_{\mathrm{P}}$ thio effect $\left(k_{\mathrm{O}} / k_{R_{\mathrm{P}}}\right)$ of 42 is observed (14). Experimentally, R69D in the absence of $\mathrm{Ca}^{2+}$ shows a 70-fold drop in the $R_{\mathrm{P}}$ thio effect $\left(k_{\mathrm{O}} / k_{R_{\mathrm{P}}}=0.6\right)$, which is partially restored $\left(k_{\mathrm{O}} / k_{R_{\mathrm{P}}}=\right.$ 9.4) upon $\mathrm{Ca}^{2+}$ addition. Together with the structural data obtained by NMR by Kravchuk et al., it was suggested that the small $R_{\mathrm{P}}$ thio effect demonstrated by R69D originates from increased flexibility in the active site, which allows the enzyme to accommodate a sulfur atom in the pro- $R$ position. Binding of $\mathrm{Ca}^{2+}$ in the R69D active site partially restores the network of interactions in this region, causing this mutant to more closely resemble the WT structurally (14). Here we compared the rmsd values of the active site residues of the WT and R69D- $\mathrm{Ca}^{2+}$ upon substrate binding. Our results show that for residues within $5 \AA$ of the docked cyclic inositol 1,2-phosphate (H32, D33, D67, D69, H82, G83, K115, E117, R163, W178, D180, D198, and Y200), the rmsd between the substrate-bound enzyme and the unbound enzyme is $3.6 \AA^{2}$ for $\mathrm{R} 69 \mathrm{D}-\mathrm{Ca}^{2+}$ while it is 0.14 $\AA^{2}$ for the WT. This is additional information supporting the fact that the decreased $R_{\mathrm{P}}$ thio effect demonstrated by R69D- $\mathrm{Ca}^{2+}$ is due to the local flexibility of the active site.

Role of His311 in $\mathrm{Ca}^{2+}$-Dependent Mammalian PI-PLCs. In the previous section, we have shown that $\mathrm{Ca}^{2+} / \mathrm{Arg} 69$ forms bidentate interactions with the $2-\mathrm{OH}$ group and the pro- $S_{\mathrm{P}}$ oxygen of the product intermediate, inositol 1,2-cyclic phosphate. This is similar to the mechanism that has been suggested for mammalian PI-PLC. However, it is known that the product intermediate readily releases from the active site of btPI-PLC but not that of mammalian PI-PLC. To understand these differences, we performed the molecular modeling study on mammalian PI-PLC in complex with the product intermediate, inositol 1,2-cyclic monophosphate. Interestingly, we found that the protonated His 311 readily forms an $\mathrm{H}$-bond $(2.7 \AA)$ with the pro- $R_{\mathrm{P}}$ oxygen of IcP. Note that the X-ray crystal structure of the mammalian PIPLC in complex with the product intermediate analogue, inositol-2-methylene-1,2-cyclic monophosphate, has been determined previously (31). It was observed that $\mathrm{Ca}^{2+}$ coordinated to both the pro- $S_{\mathrm{P}}$ and pro- $R_{\mathrm{P}}$ oxygen of the phosphate moiety. However, this bidentate interaction may be considered an artifact due to the methyl substitution at the 2-O position of inositol (in the analogue) preventing $\mathrm{Ca}^{2+}$ from forming a normal coordination to the $2-\mathrm{O}$ atom of inositol.

We speculate that it is His 311 that provides additional binding affinity toward IcP and causes the differences in product profiles between mammalian PI-PLC and btPI-PLC. This postulation is consistent with our recent experimental results on a $\mathrm{Ca}^{2+}$-dependent PI-PLC from Streptomyces antibioticus, which is homologous to mammalian PI-PLC (35). In this study, it was shown that a sulfur substitution at the pro- $R_{\mathrm{P}}$ position led to only a small decrease in the rate of hydrolysis $\left(k_{\mathrm{O}} / k_{R_{\mathrm{P}}}=47\right)$ but led to the premature release of IcP, possibly due to disruption of the H-bond between His 16 (the counterpart of His 311 ) and the pro- $R_{\mathrm{P}}$ oxygen atom.

\section{DISCUSSION}

The lack of movement in the His 32 side chain would indicate that its ability to function as the catalytic general 
base is not significantly altered. This would not affect the first step in the catalytic mechanism, and allow formation of the cyclic product. The movement of His 82 and the surrounding loop regions would have more significant consequences for two reasons. The first involves its ability to function as the catalytic general acid and to promote the release of the fatty acid chain (see Scheme 1). Movement of this amino acid side chain away from the oxygen that is to receive the donated proton would slow this step in the reaction, causing the overall reaction rate to be lower. Second, movement of this residue and the surrounding loop region appears to make the active site smaller in size. These changes would have an effect on substrate binding as well as proton transfer during catalysis. Additionally, binding of the substrate into a smaller active site would restrict the positioning of the $\mathrm{R}$ group that is being cleaved from the phosphate. Given that the two oxygen atoms on the phosphate are interacting with the active site calcium in the same manner as the mammalian system, the leaving R group would be significantly restricted in terms of the conformational freedom of the substrate as it enters the active site. However, the effect that is most significant in explaining the lowered catalytic rate cannot be determined by these studies.

Stereochemistry of R69D. As summarized by Kravchuk et al., having Arg 69 in the active site offers the ability to increase the thio effect toward $S_{\mathrm{P}}-\mathrm{DPP} \mathrm{P} \mathrm{I}$ and high stereoselectivity $\left(k_{R_{\mathrm{P}}} / K_{S_{\mathrm{P}}}=7600\right)(14-17)$. This was rationalized by the arginine residue having direct interactions with the pro-S oxygen of the substrate. On the basis of our observations of the distances produced in the inositol overlays using bound water molecules as a guide, we see that the $\mathrm{O}_{S}$ atom would be positioned closer to the $\mathrm{Ca}^{2+}$ than the $\mathrm{O}_{R}$ atom, and this rationalization is supported by our structural information.

Experimentally, the R69D mutant in the absence of $\mathrm{Ca}^{2+}$ showed a 3 order of magnitude drop in the $S_{\mathrm{P}}$ thio effect, which was then restored to proper levels upon addition of $\mathrm{Ca}^{2+}$ demonstrating that the $\mathrm{Ca}^{2+}$ ion can indeed substitute for the arginine and maintain the proper stereochemical preference in the active site. Along these same lines, the $R_{\mathrm{P}}$ thio effect was lower compared to that of the WT. This overall difference demonstrated an increase in stereoselectivity where the sulfur atom had a preference for the pro- $R$ position. Active site analysis using the overlayed structures and water positions shows that the preference for oxygen at the pro-S position and sulfur at the pro- $R$ position comes from two primary factors. The first is due to the distance of the atom in the pro-S position being closer to the $\mathrm{Ca}^{2+}$ ion. On the basis of hard-soft acid base chemistry, this closer position would preferentially be an oxygen atom over a sulfur atom. Additionally, inspection of the active site near the pro- $R$ position reveals that a more closed active site, which is caused by the inward movement of more than $1 \AA$ of His 82 , would result in stronger discrimination at the pro- $R$ position and preference for oxygen based on spatial considerations. Additionally, the fact that a water molecule is located between His 82 and the calcium ion, but not interacting with the imidazole nitrogens (NE1 is required for proton transfer and is adequately positioned to do so), demonstrates a location in the active site that has the capacity to accept the larger sulfur atom. This would provide an optimal location for the sulfur atom to be positioned over the oxygen. On the basis of this structural insight, we conclude that both the hardness of the oxygen and $\mathrm{Ca}^{2+}$ interaction at the pro-S position and the histidine ring location near the pro- $R$ position contribute to the preference for sulfur at the pro- $R$ position.

Calcium-Dependent Enzymes in Bacteria. On the basis of this structural evidence, it has been verified that R69D has indeed been effectively engineered to bind calcium in an optimal location previously occupied by arginine using a single-amino acid substitution. However, analysis of the relative rates of R69D with the WT demonstrates that the mutant enzyme is 500-fold slower in the presence of $1 \mathrm{mM}$ $\mathrm{Ca}^{2+}(14)$. There are a number of factors that have been addressed in this report that may help to explain the drop in enzymatic activity, for example, the smaller active site due to the mobility of His 82 and the molecular geometry of the $\mathrm{Ca}^{2+}$ ion requiring the use of active site waters in lieu of amino acids that are tethered to the protein backbone to provide stability as discussed in the previous section. Taken together, it is apparent that a single mutation, such as R69D, would not be advantageous for an organism to make en route to becoming $\mathrm{Ca}^{2+}$-dependent when compared to the mammalian enzyme structure. Clearly, other amino acid changes must accompany this substitution in a manner that is nonlinear (i.e., not changing one amino acid at a time to create a calcium binding site).

In a comparison of the ability of the mammalian enzyme to stabilize the calcium interaction with such an even distribution of ligands, while undergoing a change in molecular geometry at the calcium position during catalysis, it becomes apparent that a number of stabilizing factors may be involved to help the mammalian enzyme achieve this. The stretch of amino acids near the mammalian enzyme's general base histidine has an HYNY pattern (Figure 5, purple shading), where the bacterial enzyme has an HDSG pattern. Both stretches of amino acids have hydrogen bond donors and acceptors available for stabilization, but the mammalian enzyme also has the larger aromatic tyrosine positioned where the bacterial enzyme has a flexible glycine. A similar pattern is observed near the general acid location, and we see again the difference in the mammalian (HGYT) as compared to the bacterial enzyme's HHGP amino acids. Structurally, these amino acids are in loop regions that could allow for conformational changes, but the ability of the bacterial enzyme to undergo a larger conformational change is increased due to the flexible glycine residues. In contrast, the mammalian system appears to be stabilized by the larger hydrophobic amino acids in the loop region as shown in Figure 5. These changes, away from the active site, that appear to stabilize the mammalian enzyme in a manner that the bacterial enzyme lacks also make the transition from a bacterial calcium-independent enzyme to a calcium-dependent enzyme difficult to envision due to the large number of changes that must occur during the evolution process without losing enzymatic activity.

Recently, an enzyme which may provide insight into the nature of these changes has been found and characterized from $S$. antibioticus $(14,35-37)$. This enzyme is the first known natural $\mathrm{Ca}^{2+}$-dependent PI-PLC (known as saPLC) and should provide structural insights into the stepwise changes that need to be completed to make a non- $\mathrm{Ca}^{2+}{ }_{-}$ dependent enzyme not only bind calcium but also catalyze 
A

1 HUMAK

2 PAT

3 R69D

4 1PTG

5 1AOD

6 SAPL $\overline{C 1}$

7 Consensus

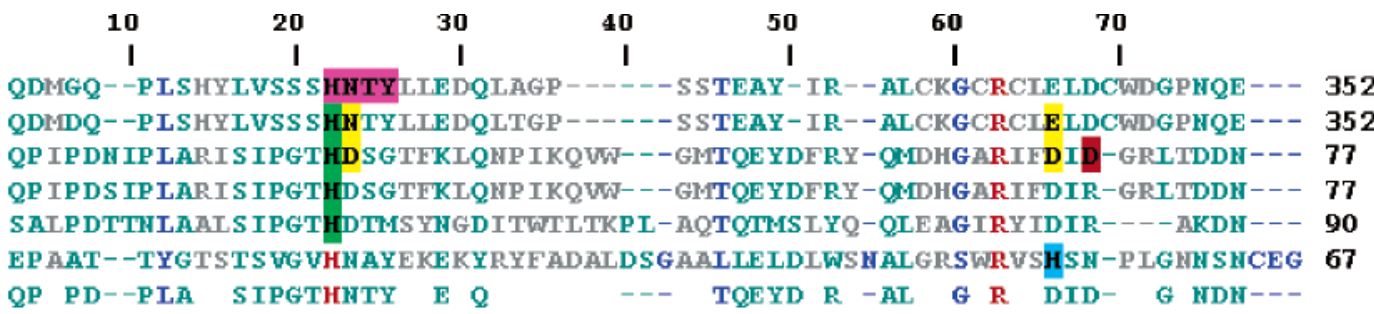
QP PD--PLA SIPGTHHTY E O

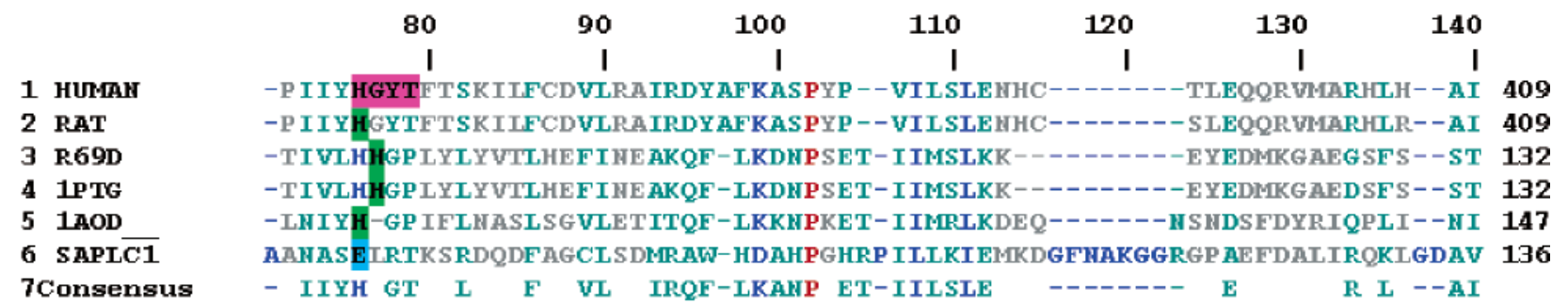
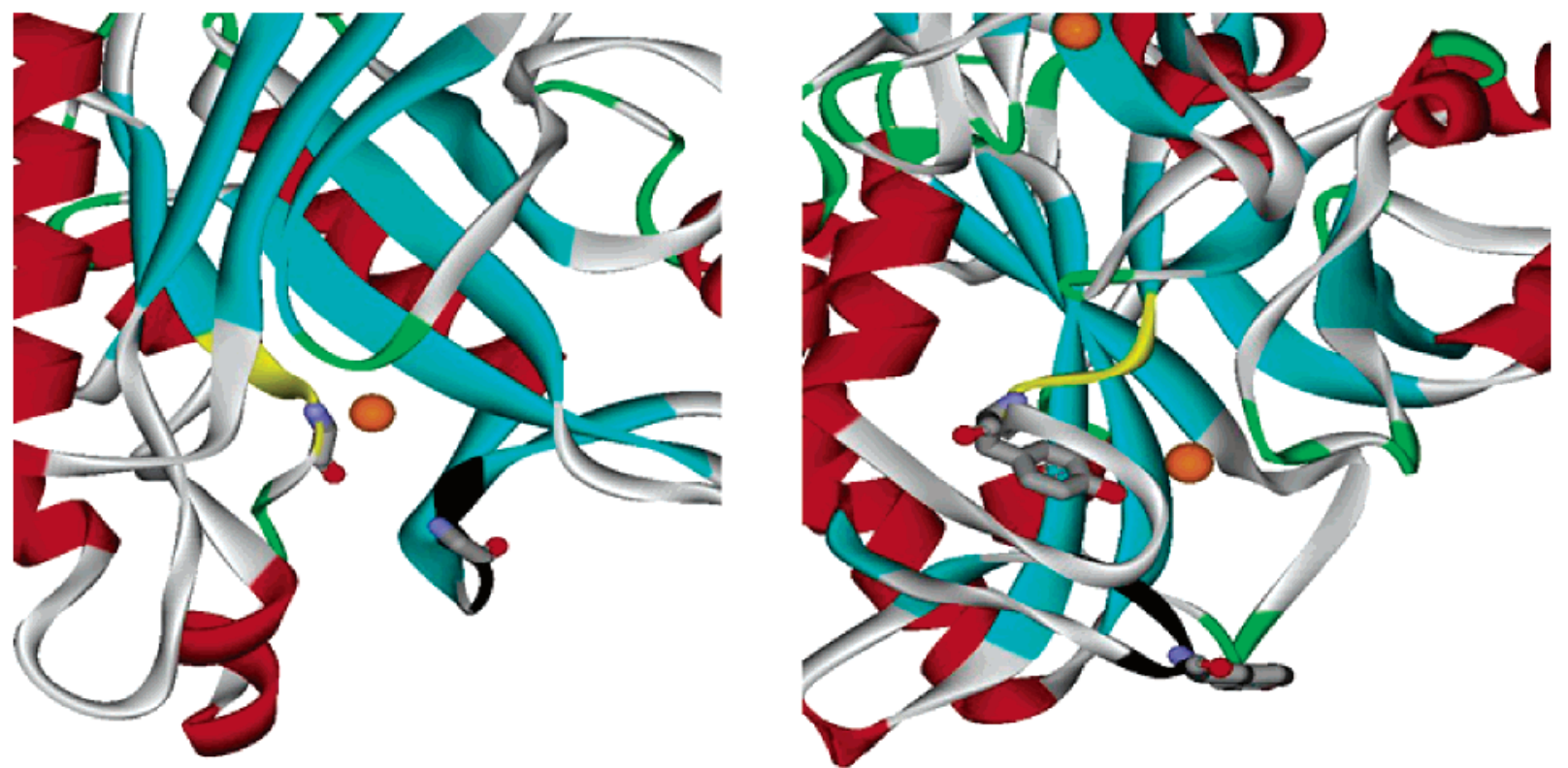

FIGURE 5: (A) Sequence alignment of the various PI-PLCs from human, rat, Bacillus (1PTG and R69D), Listeria (1AOD), and S. antibioticus (saPLC) showing regions that are conserved and important for catalysis and binding of $\mathrm{Ca}^{2+}$. Regions shaded in purple and green are the general acid-base (GA-GB) regions in the mammalian enzymes and the bacterial enzymes, respectively. The regions interacting with $\mathrm{Ca}^{2+}$ are colored yellow, and the R69D substitution position is colored red. Note that the saPLC enzyme appears to have the Ca ${ }^{2+}$ chelating amino acid in the position attributed with the GB (and vice versa) indicating a novel active site geometry as compared to the known $\mathrm{Ca}^{2+}$-dependent enzymes. (B) Active site regions of the R69D (left panel) and mammalian (right panel) enzymes demonstrating the more open bacterial and hydrophobic mammalian active sites. The mammalian tyrosine residue is shown in comparison to the bacterial enzyme's corresponding glycine as a point of reference with the yellow-colored ribbon corresponding to the cyan color in the sequence alignment.

the reaction in an efficient manner to support the needs of the organism. This enzyme has been shown to have a very high $S_{\mathrm{P}}$ thio effect and high stereoselectivity in the presence of $\mathrm{Ca}^{2+}$, indicating that there are clear mechanistic differences in the manner by which their respective reactions are carried out. After examining the structural changes that occur in the active site of the WT enzyme when compared to the R69D structure, we find that the optimal geometry around the $\mathrm{Ca}^{2+}$ ion is a major consideration for such a novel active site. After closer inspection of the amino acid sequences of the mammalian, R69D, WT, and saPLC, it appears that the saPLC may have optimized these interactions through a mechanism of amino acid juxtaposition. If one compares the amino acid alignment of the members of this family, it is seen that the general base histidine is positionally conserved among all members of bacterial PI-PLCs. The $\mathrm{Ca}^{2+}$ chelating amino acids at position 69 (in red for the R69D protein in
Figure 5) and position 67 are also conserved for all members except the saPLC enzyme. Additionally, the catalytic acid histidine (His 82) is also conserved for all members of this family. However, in each position where this conserved amino acid is expected, the oppositely conserved amino acid is observed as if the His 82 has been replaced with Asp 67. On the basis of the structural information given in this report showing the flexibility in the His 82 position and nonoptimal geometry around the calcium ion, it appears as though the saPLC active site uses the swapping of amino acid positions to adapt a calcium binding pocket and maintain a more ideal active site geometry to support efficient catalysis. To our knowledge, this is the first instance of the juxtaposition of functional amino acids that has been observed in proteins of the same enzyme class from different species. A better understanding of the effects of these changes will come with further structural studies on the saPLC enzyme. 


\section{SUPPORTING INFORMATION AVAILABLE}

Coordination distances at the active sites of 1PTG, R69D, and 1DJX upon substrate binding. This material is available free of charge via the Internet at http://pubs.acs.org.

\section{REFERENCES}

1. Rhee, S. G. (2001) Regulation of phosphoinositide-specific phospholipase C, Annu. Rev. Biochem. 70, 281-312.

2. Rebecchi, M. J., et al. (2000) Structure, function, and control of phosphoinositide-specific phospholipase C, Physiol. Rev. 80 (4), $1291-1335$

3. Meldrum, E. P. P., and Carozzi, A. (1991) The PtdIns-PLC superfamily and signal transduction, Biochim. Biophys. Acta 1092 (1), 49-71.

4. Bruzik, K. S., et al. (1994) Toward the mechanism of phosphoinositide-specific phospholipases C, Bioorg. Med. Chem. 2 (2), 4972.

5. Griffith, O. H., et al. (1999) Bacterial phosphatidylinositol-specific phospholipase C: Structure, function, and interaction with lipids, Biochim. Biophys. Acta 1441 (2-3), 237-254.

6. Miyawaki, A. (2003) Visualization of the spatial and temporal dynamics of intracellular signaling, Dev. Cell 4 (3), 295-305.

7. Essen, L. O., et al. (1996) Crystal structure of a mammalian phosphoinositide-specific phospholipase C $\delta$, Nature 380 (6575), 595-602.

8. Essen, L. O., et al. (1997) A ternary metal binding site in the C2 domain of phosphoinositide-specific phospholipase C- $\delta 1$, Biochemistry 36 (10), 2753-2762.

9. Kohda, D., et al. (1993) Solution Structure of the Sh3 Domain of Phospholipase C- $\gamma$, Cell 72 (6), 953-960.

10. Ferguson, K. M., et al. (1995) Structure of the High-Affinity Complex of Inositol Trisphosphate with a Phospholipase-C Pleckstrin Homology Domain, Cell 83 (6), 1037-1046.

11. Grobler, J. A., et al. (1996) C2 domain conformational changes in phospholipase C- $\delta 1$, Nat. Struct. Biol. 3 (9), 788-795.

12. Moser, G. B., Jr., Meyer, J. E., Chakraborty, T., Wehland, J., and Heinz, D. W. (1997) Crystal structure of the phosphatidylinositolspecific phospholipase C from the human pathogen Listeria monocytogenes, J. Mol. Biol. 17 (273), 269-282.

13. Gassler, C. S., Rysn, M., Liu, T., Griffith, O. H., and Heinz, D. W. (1997) Probing the roles of active site residues in phosphatidylinositol-specific phospholipase $\mathrm{C}$ from Bacillus cereus by sitedirected mutagenesis, Biochemistry 36 (42), 12802-12813.

14. Kravchuk, A. V., et al. (2003) Engineering a catalytic metal binding site into a calcium-independent phosphatidylinositol-specific phospholipase $\mathrm{C}$ leads to enhanced stereoselectivity, Biochemistry 42 (8), 2422-2430.

15. Kravchuk, A. V., et al. (2001) Mechanism of phosphatidylinositolspecific phospholipase C: Origin of unusually high nonbridging thio effects, Biochemistry 40 (18), 5433-5439.

16. Hondal, R. J., et al. (1997) Phosphatidylinositol-specific phospholipase C: Kinetic and stereochemical evidence for an interaction between arginine- 69 and the phosphate group of phosphatidylinositol, Biochemistry 36 (22), 6633-6642.

17. Hondal, R. J., et al. (1998) Mechanism of phosphatidylinositolspecific phospholipase C: A unified view of the mechanism of catalysis, Biochemistry 37 (13), 4568-4580.

18. Minor, Z. W. (1997) Processing of X-ray Diffraction Data Collected in Oscillation Mode, Methods Enzymol. 276 (A), 307326.

19. A.Vagin, A. T. (1997) MOLREP: An automated program for molecular replacement, J. Appl. Crystallogr. 10, 1022-1025.

20. Potterton, E., Briggs, P., Turkenburg, M., and Dodson, E. (2003) A graphical user interface to the $\mathrm{CCP} 4$ program suite, Acta Crystallogr. D59, 1131-1137.
21. Heinz, D. W., Ryan, M., Bullock, T. L., and Griffith, O. H. (1995) Crystal structure of the phosphatidylinositol-specific phospholipase $\mathrm{C}$ from Bacillus cereus in complex with myoinositol, EMBO J. 14 (16), 3855-3863.

22. Brunger, A. T., Adams, P. D., Clore, G. M., DeLano, W. L., Gros, P., Grosse-Kunstleve, R. W., Jiang, J. S., Kuszewski, J., Nilges, M., Pannu, N. S., Read, R. J., Rice, L. M., Simonson, T., and Warren, G. L. (1998) Crystallography \& NMR system: A new software suite for macromolecular structure determination, Acta Crystallogr. D54 (5), 905-921.

23. Murshudov, G. N., Vagin, A. A., Lebedev, A., Wilson, K. S., and Dodson, E. J. (1999) Efficient anisotropic refinement of macromolecular structures using FFT, Acta Crystallogr. D55 (1), 247255.

24. Jones, T. A., Zou, J. Y., Cowan, S. W., and Kjeldgaard, M. (1991) Improved methods for building protein models in electron density maps and the location of errors in these models, Acta Crystallogr. A47, 110-119.

25. Morris, R. J., Perrakis, A., and Lamzin, V. S. (2002) ARP/wARP's model-building algorithms. I. The main chain, Acta Crystallogr. D58, 968-975.

26. Laskowski, R. A., MacArthur, M. W., Moss, D. S., and Thornton, J. M. (1993) PROCHECK: A program to check the stereochemical quality of protein structures, J. Appl. Crystallogr. 26, 283291.

27. Morris, A. L., MacArthur, M. W., Hutchinson, E. G., and Thornton, J. M. (1992) Stereochemical quality of protein structure coordinates, Proteins 12, 345-364

28. Lu, G. (2000) TOP: A new method for Protein Structure Comparisons and Similarity Searches, J. Appl. Crystallogr. 33, 176-183.

29. Kubiak, R. J., et al. (2001) Involvement of the Arg-Asp-His catalytic triad in enzymatic cleavage of the phosphodiester bond, Biochemistry 40 (18), 5422-5432.

30. Ellis, M. V., et al. (1998) Catalytic domain of phosphoinositidespecific phospholipase C (PLC). Mutational analysis of residues within the active site and hydrophobic ridge of plcdelta1, J. Biol. Chem. 273 (19), 11650-11659.

31. Essen, L. O., et al. (1997) Structural mapping of the catalytic mechanism for a mammalian phosphoinositide-specific phospholipase C, Biochemistry 36 (7), 1704-1718.

32. Grobler, J. A., et al. (1998) Catalysis by phospholipase C $\delta 1$ requires that $\mathrm{Ca}^{2+}$ bind to the catalytic domain, but not the $\mathrm{C} 2$ domain, Biochemistry 37 (14), 5020-5028.

33. Rebecchi, M. J., and Rosen, O. M. (1987) Purification of a phosphoinositide-specific phospholipase C from bovine brain, $J$. Biol. Chem. 262 (26), 12526-12532.

34. Heinz, D. W., et al. (1998) Structural and mechanistic comparison of prokaryotic and eukaryotic phosphoinositide-specific phospholipases C, J. Mol. Biol. 275 (4), 635-650.

35. Zhao, L., et al. (2003) A novel calcium-dependent bacterial phosphatidylinositol-specific phospholipase $\mathrm{C}$ displaying unprecedented magnitudes of thio effect, inverse thio effect, and stereoselectivity, J. Am. Chem. Soc. 125 (1), 22-23.

36. Iwasaki, Y., Nakano, H., and Yamane, T. (1994) Phospholipase D from Streptomyces antibioticus: Cloning, sequencing, expression, and relationship to other phospholipases, Appl. Microbiol. Biotechnol. 42 (2), 290-299.

37. Iwasaki, Y., Tsubouchi, Y., Ichihashi, A., Nakano, H., Kobayashi, T., Ikezawa, H., and Yamane, T. (1998) Two distinct phosphatidylinositol-specific phospholipase Cs from Streptomyces antibioticus, Biochim. Biophys. Acta 1391 (1), 52-66.

BI047896V 